\title{
Types of Dyslipidemia in Type 2 DM Patients of Bhubaneswar region
}

\author{
Rajendra Dev Bhatt, ${ }^{1 *}$ Kamal Lochan ${ }^{2}$
}

BACKGROUND: A characteristic pattern, termed dyslipidemia, consists of deranged of any single components of lipid profile test. This pattern is most frequently seen in diabetes and may be a preventable risk factor for subsequent cardiovascular disease. This study determined the influence of type 2 diabetes mellitus (T2DM) on lipid profile of diabetic patients reporting in a tertiary hospital in Bhubaneswar, India.

METHODS: 50 confirmed T2DM patients and 50 non-diabetic control subjects were selected for the study. Fasting and 2 hours post prandial blood samples were collected from both study and control patients. Fasting blood sample was analyzed for lipid profile test and serum glucose, and post prandial sample was analyzed for serum glucose only.

RESULTS: Sixty two (62\%) of diabetic patients were males whilst thirty eight $(38 \%)$ were females in this study. The mean plasma glucose levels, Total cholesterol and Triacylglycerol were significantly raised in the diabetics as compared to those in the control subjects. This is substantiated by the fact that the entire lipid fractions are disturbed in diabetics as compared to healthy controls.

CONCLUSION: Thus dyslipidemia was quite common in diabetes and Hypertriglyceridemia was the most common one.

Keywords: Type-2 Diabetes Mellitus, Dyslipidemia, Lipid Profile, Triglyceride, HDL-C, LDL-C, Total Cholesterol.

\section{(c) 2015 Nepalese Association for Clinical Chemistry}

\section{Introduction}

Diabetes mellitus (DM) is a syndrome consisting of metabolic, vascular and neuropathic components that are interrelated. It is defined as group of metabolic disorder that is characterized by hyper-glycemia resulting from defect in insulin secret-ion, insulin action or both. The lack of effective insulin action leads to alteration in carbohydrate, fat and protein metabolism [1]. T2DM is caused by relatively impaired insulin secretion and peripheral insulin resistance [2].

Diabetes is no more an epidemic but it has it has been turned into a global pandemic. Diabetes has been recognized as a health threat worldwide. As per the global projection by international diabetes foundation the number of diabetic patients has risen sharply in recent years. While in 1985, thirty million people had diabetes worldwide; the number rose to one hundred million in 2000, two hundred eighty five million in 2010 and is estimated to be four hundred thirty five million, $7.8 \%$ of the adult world population, by 2030 [3].

According to WHO, $70 \%$ of current cases of diabetes, occur in developing countries [4]. Among these India has the world's largest population with an estimated 50.8 million people living with diabetes [3]. The international journal of diabetes for developing countries has declared India as the diabetes capital of the world [5].

A characteristic pattern, termed dyslipidemia, consists of increased triglycerides (TAG), Total cholesterol (TC), low density lipoprotein (LDL), and very low density lipoprotein (VLDL) cholesterol and decreased high density lipoprotein (HDL). This pattern is most frequently seen in diabetes and may be a preventable risk factor for subsequent cardiovascular disease.

Patients with T2DM are at greater risk of developing vascular diseases because of lipid changes. Lipid abnormalities and insulin use is critically discussed in diabetics [6]. The most typical lipoprotein pattern reported in diabetes, also known as diabetic dyslipidemia or atherogenic dyslipidemia consists of moderate elevation in TC, TAG and LDL-Cholesterol levels with or without low HDL-Cholesterol levels in blood.

The degree of variations in lipid profile of diabetic patients may not generalized to all region and should be individualized to specific regions as ethnic, hereditary and environmental factors influence lipid profile. Due to increasing cardiovascular problems in T2DM patients [7], this study was conducted to observe the co-

\footnotetext{
'Department of Biochemistry, Hi-Tech Medical College and Hospital, Bhubaneswar, Odisha, India.

Correspondence to: Rajendra Dev Bhatt, Department of Biochemistry, Kathmandu University Hospital-Dhulikhel Hospital, Dhulikhel. E-mail: bhattdev.rajendra@gmail.com
} 
relation of T2DM and types of lipid abnormalities in T2DM. Thus this research aims to evaluate the types of dyslipidemia in T2DM patients of Bhubaneswar region.

\section{Methods}

The study was carried out on the patients of medical OPD at Hi-Tech Medical College and Hospital in Bhubaneswar. 50 T2DM patients were recruited after their consent had been sought.

The study targeted T2DM patients, medically diagnosed by American Diabetes Association (ADA) criteria. Randomly selected age and sex matched individuals, with no history of diabetes or any type of illness and not on statins were used as controls.

Patients with type 1 DM, other ailments, metabolic disorders and other causes of hyperlipidemia were not included in this study. Pregnant women, patients on statins for abnormal lipid treatment (both for T2DM and controls) were also excluded.

Venous blood samples were taken from both diabetic and control patients at overnight fasting and 2 hours post prandial state. Investigations carried out were, blood glucose fasting and $2 \mathrm{hrs}$ post prandial blood glucose, and fasting lipid profile including TC, TAG, HDL-C, LDL- C and very low density lipoprotein cholesterol (VLDL-C).

Serum TC was determined by an enzymatic (CHOD/PAP) colorimetric method and TAG was determined by an enzymatic (GPO-PAP) method [8]. HDL-C was estimated by a precipitant method and LDL-C by was estimated by using Friedewald's formula as shown below:

LDL-C = TC - HDL-C $-($ TAG/5), where TAG/5 is approximately equal to VLDL-C.

Serum glucose was determined by using the glucose oxidase/per oxidase enzymatic method [2].

Dyslipidemia was defined using the National Cholesterol Education Programme - Adult Treatment Panel III (NCEP - ATP III) (National Cholesterol Education Programme, 2002) criteria as shown in Table 1.
Table 1. ATP III Classification of LDL-C, TC, HDL-C and TG (mg/dL).

\begin{tabular}{ll}
\hline LDL-Cholesterol & \\
\hline$<100$ & Optimal \\
$100-129$ & Near optimal/ above optimal \\
$130-159$ & Borderline high \\
$160-189$ & High \\
$>190$ & Very high \\
\hline Total Cholesterol & \\
\hline$<200$ & Desirable \\
$200-239$ & Borderline high \\
$>240$ & High \\
\hline HDL Cholesterol & \\
\hline$<40$ & Low \\
$>60$ & High \\
\hline Triacylglycerol & \\
\hline$<150$ & Normal \\
$150-199$ & Borderline high \\
$200-499$ & High \\
$>500$ & Very high \\
\hline
\end{tabular}

\section{Results}

In this study 50 diagnosed cases of T2DM were observed as cases and 50 non diabetic were observed as controls. There was significant difference in the fasting blood sugar, post prandial blood sugar level and lipid profile test in control and T2DM patients.

The mean serum TG in our study was $188.9 \pm 69.07 \mathrm{mg} / \mathrm{dL}$ as compared to $117.56 \pm$ $23.51 \mathrm{mg} / \mathrm{dL}$ of controls. The mean serum total cholesterol was $203.38 \pm 48.0 \mathrm{mg} / \mathrm{dL}$ as compared to $161.4 \pm 24.55 \mathrm{mg} / \mathrm{dL}$ of controls. While HDLC was $38.22 \pm 5.85 \mathrm{mg} / \mathrm{dL}$ as compared to $43.46 \pm 6.95 \mathrm{mg} / \mathrm{dL}$ of controls, which was significantly lower to that of controls as shown in Table 2.

\section{Discussion}

We found significant difference $(\mathrm{P}<0.0001)$ at the Triglyceride level in control and cases where mean \pm S.D is $117.56 \pm 23.51$ and 188.9 \pm 69.07 of control and cases respectively. We also found $74 \%$ diagnosed diabetics have $>150 \mathrm{mg} / \mathrm{dl}$, $14 \%$ have $>250 \mathrm{mg} / \mathrm{dl}$ and $10 \%$ have $>300 \mathrm{mg} / \mathrm{dl}$ of Triglyceride out of 50 cases. And only 6\% control have $>150 \mathrm{mg} / \mathrm{dl}$ of Triglyceride.

We also observe significant difference $(\mathrm{p}<0.001)$ in the Total cholesterol level, where mean \pm S.D is $161.4 \pm 24.55$ and $203.38 \pm 48.00$ of control and cases respectively. 
Table 2. Fasting and post prandial serum glucose of type 2 diabetic patients and controls

\begin{tabular}{lcccc}
\hline Parameters & Patients & Controls & P value & T \\
\hline Fasting serum glucose $(\mathrm{mg} / \mathrm{dL})$ & $144.62 \pm 71.61$ & $85.86 \pm 9.33$ & $<0.0001$ & 5.75 \\
Post prandial serum glucose $(\mathrm{mg} / \mathrm{dL})$ & $209.60 \pm 75.49$ & $113.88 \pm 15.73$ & $<0.0001$ & 8.78 \\
Triacylglycerol $(\mathrm{mg} / \mathrm{dL})$ & $188.9 \pm 69.07$ & $117.56 \pm 23.51$ & $<0.0001$ & 6.91 \\
Total Cholesterol (mg/dL) & $203.38 \pm 48.0$ & $161.4 \pm 24.55$ & $<0.0001$ & 5.51 \\
HDL $(\mathrm{mg} / \mathrm{dL})$ & $38.22 \pm 5.85$ & $43.46 \pm 6.95$ & $<0.0001$ & 4.07 \\
LDL $(\mathrm{mg} / \mathrm{dL})$ & $127.36 \pm 45.19$ & $96.32 \pm 42.90$ & $<0.0001$ & 4.25 \\
VLDL $(\mathrm{mg} / \mathrm{dL})$ & $38.90 \pm 15.73$ & $23.28 \pm 4.70$ & $<0.0001$ & 6.72 \\
\hline
\end{tabular}

We also found $48 \%$ of Diabetic have $>200 \mathrm{mg} / \mathrm{dl}$ of Total Cholesterol from 50 cases of diagnosed diabetics, while only $4 \%$ control have $>200 \mathrm{mg} / \mathrm{dl}$ of Total cholesterol.

Smith S. and Lall A. M also found mean \pm SD of Total cholesterol $299.36+13.46$ in their study in Allahabad, India [5].

A significant difference in the HDL and LDL cholesterol level in control and cases. Where mean \pm S.D is $43.46 \pm 6.95$ and $38.22 \pm 5.86$ of HDL and is $96.32 \pm 42.90$ and $127.36 \pm 45.19$ of LDL in control and cases respectively. P value of HDL and LDL is $<0.0001$ which means there is significant difference between control and cases. Among 50 of diabetics $38 \%$ have $<35 \mathrm{mg} / \mathrm{dl}$ of HDL and $8 \%$ out of 50 controls have $<35 \mathrm{mg} / \mathrm{dl}$ of HDL Cholesterol.

Another study done by Rakesh et al [9] most common pattern was combined dyslipidemia with high LDL and low HDL in both males $(22.7 \%)$ and females (33\%).

In our study, level of TC, TAG, VLDL-C \& LDL-C were significantly increased $(\mathrm{p}<0.001)$ while HDL-C level was significantly decreased $(\mathrm{p}<0.01)$ in T2DM. These findings are consistent with those of Taha D et al (2002) [10], Howard BV et al (1999) [11], O'Neal DN (1998) [12], Mazanto et al (1993) [13]. Niemeijer et al [14] and they found that increased TAG \& decreased HDL-C plasma concentration are common features of dyslipidemia in T2DM. Oki JC in
1995 [15] stated that essentially any dyslipidemic pattern can be present.

In a study by $H$. Surekha Rani et al [16] an attempt has been made to evaluate the risk factors for coronary heart disease in DM patients. It is observed that, TC, VLDL, LDLs, TGs were high and the levels of HDLs were low compared to controls.

A significant difference was noticed in the VLDL Cholesterol level in control and cases where mean \pm S.D is $23.84 \pm 4.70$ and $38.90 \pm 15.73$ of control and cases respectively with $\mathrm{p}$ value $<0.001$.

\section{Conclusion}

T2DM patients in this study had elevated levels of TAG, TC with slightly elevated levels of LDL$\mathrm{C}$ and reduced levels of HDL-C. This indicates the influence of T2DM on abnormal lipid profile of patients with its associated danger of elevated CVD risk.

Diabetic patients with complication tend to have higher levels of lipid fractions (TAG, T. Chol, LDL and VLDL) and lower level of HDL. This suggests that there appears to be some relation between the geneses of various vascular complications in the presence of lipid abnormality. So it is important to aim at critical control of diabetes mellitus to prevent or at least postpone the onset of various hyperlipidemia related complications.

\section{REFERENCES}

1. Gavin JR. Report of the expert committee on the diagnosis and classification of diabetes mellitus. Diabetes Care 1998; 21(1): 5-519.

2. TIETZ Text Book of Clinical Chemistry \& molecular diagnostics 200642

3. Mishra A. and Mittal N. 2010. Diabetes: Concerns for India and the latest cures. WDF 2010.
4. World Health Organization. Diabetes mellitus, (WHO Tech Rep Series). WHO, Geneva, 1985, 1997.

5. Smith S. and Lall A. M. 2008. A Study on Lipid Profile Levels of Diabetics and Non-Diabetics Among Naini Region of Allahabad, India. Turk J Biochem 2008:33 (4): 138-141.

6. Mitka M; Aggressive lipid, hypertension targeting yields no benefit for some with diabetes.
JAMA, 2010; 303(17): 16811683Genetic Association between Insulin Resistance and Total Cholesterol in Type 2 Diabetes Mellitus - A preliminary observation. Online J Health Allied Scs. 2005;1:4.

7. Genetic Association between Insulin Resistance and Total Cholesterol in Type 2 Diabetes Mellitus - A preliminary observation. Online J Health Allied Scs. 2005;1:4. 
8. Godkar P and Godkar D (2005) Text book of medical laborarty technology.Ed.2 chemistry of lipid (Bhalani publishing house) New Delhi-India

9. Rakesh M Parikh, Sashank R Joshi, Padmavathy S, Menon, Nalini S, Shash (2010). Prevalence and Pattern of Diabetic Dyslipidemia in Indian type 2 Diabetic patients. Diabetes and Metabolic Syndrome; Clinical Research and Review 4(1):10-12.

10. Taha D. Hyperlipidemia in children with type 2 DM. J Pediatr
Endocrinol Metab. 15 Suppl; 1 : 05 507, Apr 2002.

11. Howard B.V. Insulin resistance and lipid metabolism. Am J Cardiol. 84(1A):28J-32J, Jul 81999.

12. O'neal D.N. et al. Lipid levels and peripheral vascular disease in diabetics and non diabetic subjects. Atherosclerosis.146 (1):1-8, Jan 1998.

13. E. Mazanto, Zambon A., Lapolla A., Zambon S.,Braghetto L., Crepaldi G. and Fedele D : Diabetes Care.16(2):469-475,1993.
14. Niemeijer-Kanters S.D. et al. Dyslipidemia in diabetes mellitus. Ned Tijd schr Geneeskd. 145(16) :769-774,Apr,21,2001.

15. Oki J.C. Dyslipidemias in patients with diabetes mellitus. Classification and risks and benefits of therapy. Pharmacotherapy 15(3):317-337, May-June1995.

16. H. Surekha Rani., G. Madhavi., V Ramachandra Rao., B.K.Sahay and A. Jyothy. Risk Factors for Coronary Heart Disease in Type 2 DM. Indian Journal of Clinical Biochemistry 2005; 20 (2): 75-80. 